\title{
Novel xenon calibration scheme for two-photon absorption laser induced fluorescence of hydrogen
}

Drew Elliott

Earl Scime

Zachary Short

\section{Digital Commons Citation}

Elliott, Drew; Scime, Earl; and Short, Zachary, "Novel xenon calibration scheme for two-photon absorption laser induced fluorescence of hydrogen" (2016). Faculty \& Staff Scholarship. 252.

https://researchrepository.wvu.edu/faculty_publications/252

This Article is brought to you for free and open access by The Research Repository @ WVU. It has been accepted for inclusion in Faculty \& Staff Scholarship by an authorized administrator of The Research Repository @ WVU. For more information, please contact beau.smith@mail.wvu.edu. 


\title{
Novel xenon calibration scheme for two-photon absorption laser induced fluorescence of hydrogen
}

\author{
Drew Elliott, Earl Scime, and Zachary Short ${ }^{\mathrm{a})}$ \\ Department of Physics and Astronomy, West Virginia University, Morgantown, West Virginia 26056, USA
}

(Presented 8 June 2016; received 31 May 2016; accepted 20 June 2016;

published online 14 July 2016)

Two photon absorption laser induced fluorescence (TALIF) measurements of neutral hydrogen and its isotopes are typically calibrated by performing TALIF measurements on krypton with the same diagnostic system and using the known ratio of the absorption cross sections [K. Niemi et al., J. Phys. D 34, 2330 (2001)]. Here we present the measurements of a new calibration method based on a ground state xenon scheme for which the fluorescent emission wavelength is nearly identical to that of hydrogen, thereby eliminating chromatic effects in the collection optics and simplifying detector calibration. We determine that the ratio of the TALIF cross sections of xenon and hydrogen is $0.024 \pm 0.001$. Published by AIP Publishing. [http://dx.doi.org/10.1063/1.4955489]

\section{INTRODUCTION}

The neutral density at the plasma edge affects global quantities in tokamak plasmas such as the threshold for the $\mathrm{L}$ to $\mathrm{H}$ transition and the level of turbulence in the edge. ${ }^{1,2}$ Penetration by neutrals also plays an important role in cross field transport of particles and energy in fusion plasmas. ${ }^{3}$ Twophoton absorption laser induced fluorescence (TALIF) has been developed as a method to measure both the temperature and absolute density of atomic hydrogen and its isotopes in plasma. ${ }^{4,5}$

Fast pressure gauges and Balmer-alpha emission spectroscopy are two methods used to measure the density and temperature of neutral populations within hot hydrogen plasmas. ${ }^{6,7}$ Pressure gauges intrinsically convolve neutral temperature and density and are incapable of non-perturbatively measuring the neutral density profile. Line-integrated Balmer-alpha measurements are inverted to obtain profile measurements of the density of the $n=3$ excited state of atomic hydrogen and its isotopes. A collisional radiative (CR) model is then used to determine the total neutral density profile from the inverted emission measurements. ${ }^{8}$ A direct TALIF measurement of the neutral density eliminates the uncertainties introduced by the CR model and the need for the electron temperature and density measurements.

For fusion edge plasma conditions, the ground state density of hydrogen and its isotopes is a reliable measure of the overall atomic density. ${ }^{9}$ TALIF directly measures the density of a hydrogenic species ground state by exciting the $n=1$ to the $n=3$ state (transition energy of $12.096 \mathrm{eV}$ ) and measuring the intensity of emission from the $n=3$ to $n=2$ state at $656.3 \mathrm{~nm} .{ }^{4,5,10,11}$ For TALIF to produce an absolute density measurement, the diagnostic is calibrated using a known density of a gas whose relative two-photon absorption cross sec-

\footnotetext{
Note: Contributed paper, published as part of the Proceedings of the 21st Topical Conference on High-Temperature Plasma Diagnostics, Madison, Wisconsin, USA, June 2016.

a) Author to whom correspondence should be addressed. Electronic mail: zdshort@mix.wvu.edu
}

tion to hydrogen is known and for which the initial transition is accessible with the same laser. The calibration gas commonly used for hydrogen TALIF calibration is krypton., ${ }^{4,5,10,11}$ The krypton calibration scheme begins with a transition from the ground state to the $5 \mathrm{p}$ excited state (transition energy 12.143 $\mathrm{eV}$ ) and the fluorescence occurs at $826 \mathrm{~nm}$. While the initial transitions for hydrogen and krypton are nearly identical, the fluorescing wavelengths are quite different, necessitating a detailed understanding of the performance of the photodetector across a wide spectral range, different emission line filters, and accounting for wavelength dependent effects in the light collection system. The wavelength dependent optical effects are particularly problematic in the light collection schemes with long path lengths.

Here we present TALIF measurements of ground state xenon using a new scheme that is ideally suited for calibrating hydrogen TALIF systems. The xenon ground state is a $J=0,5 p^{6}$ state with an ionization energy of $12.130 \mathrm{eV}$. Lying $11.848 \mathrm{eV}$ above the ground state is the $5 p^{5}\left({ }^{2} P_{3 / 2}^{\mathrm{o}}\right) 7 f$ state with $J=2$. This energy difference is equivalent to two $209.287 \mathrm{~nm}$ photons. Single photon decay primarily occurs from the $7 f$ state to either the $5 p^{5}\left({ }^{2} P_{1 / 2}^{\mathrm{o}}\right) 6 s(J=1)$ state via a $544.0 \mathrm{~nm}$ photon or the $5 p^{5}\left({ }^{2} \mathrm{P}_{3 / 2}^{\mathrm{o}}\right) 5 d$ state via a $656.0 \mathrm{~nm}$ photon. ${ }^{12}$ Xe TALIF schemes previously explored employ very different pump and fluorescence wavelengths $(224.26 \mathrm{~nm}$ and $835 \mathrm{~nm}$, respectively). ${ }^{13}$

\section{EXPERIMENTAL APPARATUS}

This TALIF system begins with a Spectra-Physics Quanta-Ray Pro-270 Nd:YAG laser with a maximum power of $750 \mathrm{~mJ} /$ pulse at $532 \mathrm{~nm}$ at a repetition rate of $20 \mathrm{~Hz}$. The dye laser is a Sirah CobraStretch optimized for $615 \mathrm{~nm}$ light with a mixture of Rhodamine B and Rhodamine 101 dyes. The output energy of the dye laser is $\sim 100 \mathrm{~mJ} /$ pulse and the pulse width is $12 \mathrm{~ns}$. The output of the dye laser is frequency doubled to $306-315 \mathrm{~nm}$ in a SHG-250 BBO crystal, mixed back with the $615 \mathrm{~nm}$ fundamental, and passed through a SHG-206 BBO crystal to generate a third harmonic beam at 204-209 nm. The 


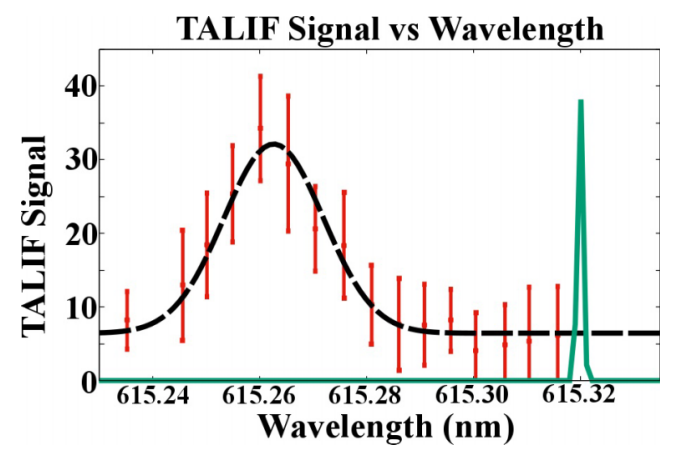

FIG. 1. Typical deuterium TALIF spectrum obtained in a spheromak.

$205 \mathrm{~nm}$ light has a typical power of $1 \mathrm{~mJ} /$ pulse, a temporal FWHM of $7 \mathrm{~ns}$, an energy stability of 5\%-7\%, and a spatial profile with a diameter of $5 \mathrm{~mm}$. The measured line width of the $615 \mathrm{~nm}$ beam is $\sim 0.1 \mathrm{~cm}^{-1}$, which should result in a similar line width, as per the design specifications, at $205 \mathrm{~nm}$.

The collected light is filtered with a bandpass filter and focused onto a photomultiplier tube (PMT) (Hamamatsu H11526-20-NF). The PMT signal is then processed by a Stanford Research Systems Fast Gated Integrator and Boxcar Averager (Model SR250). The boxcar acquires the integrated output of the PMT over a $50 \mathrm{~ns}$ interval synchronized to the laser pulse, averages 10 such measurements, and outputs a voltage proportional to the integrated signal intensity. The result is recorded along with the measured wavelength of the $\sim 615 \mathrm{~nm}$ beam, which is obtained with a High Finesse WS7 wavelength meter accurate to $0.075 \mathrm{pm}$. The laser is stepped in wavelength over a spectral range and several laser pulses are taken at each wavelength. A deuterium TALIF spectrum obtained in a spheromak with this TALIF system is shown in Figure $1 .{ }^{14}$

The TALIF emission, $S(\lambda)$, from neutrals of velocity space density $n(v)$ is

$$
S(\lambda)=\frac{\Delta \Omega}{4 \pi} n(v) I^{2} \sigma \alpha G
$$

proportional to the square of the laser intensity, I. $\Delta \Omega$ is the solid angle over which the emitted light is collected, $\sigma$ is the absorption cross section from the initial state to the excited state, and $\alpha$, which has a value between 0 and 1 , accounts for the branching ratio from the excited state to the final state of

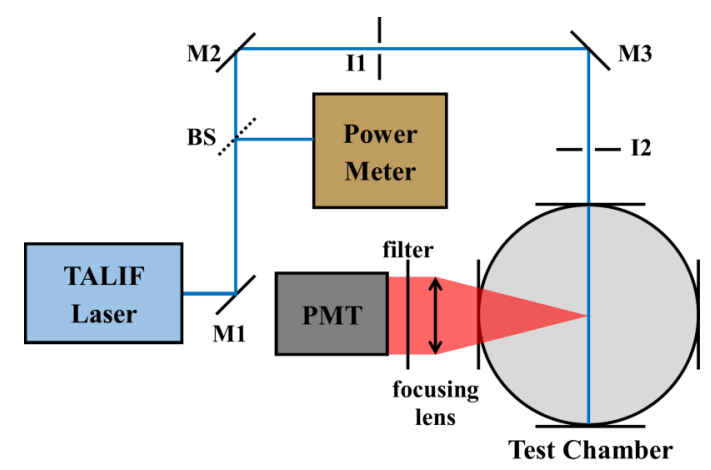

FIG. 2. Optical configuration used for these experiments. BS: beam splitter; I1 and I2: irises; M1-M3: mirrors.
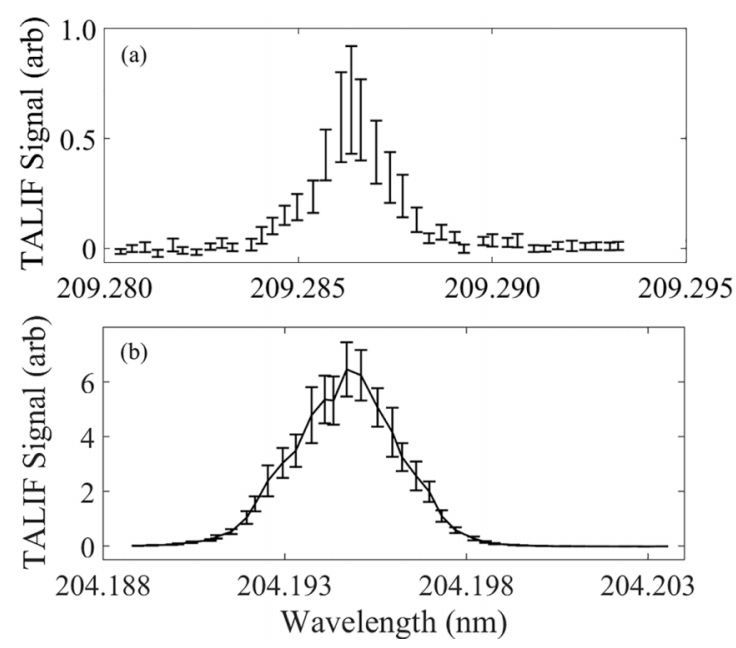

FIG. 3. (a) Xenon TALIF spectrum. (b) Krypton TALIF spectrum.

the fluorescent transition, the transmission efficiency of any optical filters, and the quantum efficiency of the photodetector. $G$ is the overall gain of the photodetector. The known ratio of cross sections ${ }^{10}$ between the krypton and the deuterium schemes, $\left(\sigma_{D e u} / \sigma_{K r}\right)$, is 0.62 .

Because the objective of these experiments was to establish the relative absorption cross sections for krypton and xenon, and thereby determine a value for the relative cross section value between xenon and hydrogen, the measurements were performed in a simple, low pressure, gas filled chamber. A diagram of the experimental configuration is shown in Figure 2. To avoid saturation effects and minimize optical complexity, the injected laser beam was not focused. Fluorescent light collection was accomplished through a $1 \mathrm{in}$. diameter, $15 \mathrm{~cm}$ focal lens oriented perpendicular to the injected laser beam. The fluorescent emission from krypton was filtered with a $10 \mathrm{~nm}$ wide bandpass filter centered on $830 \mathrm{~nm}$ and the xenon emission was filtered with the same bandpass filter, $656 \pm 1.0 \mathrm{~nm}$, used for hydrogen TALIF.
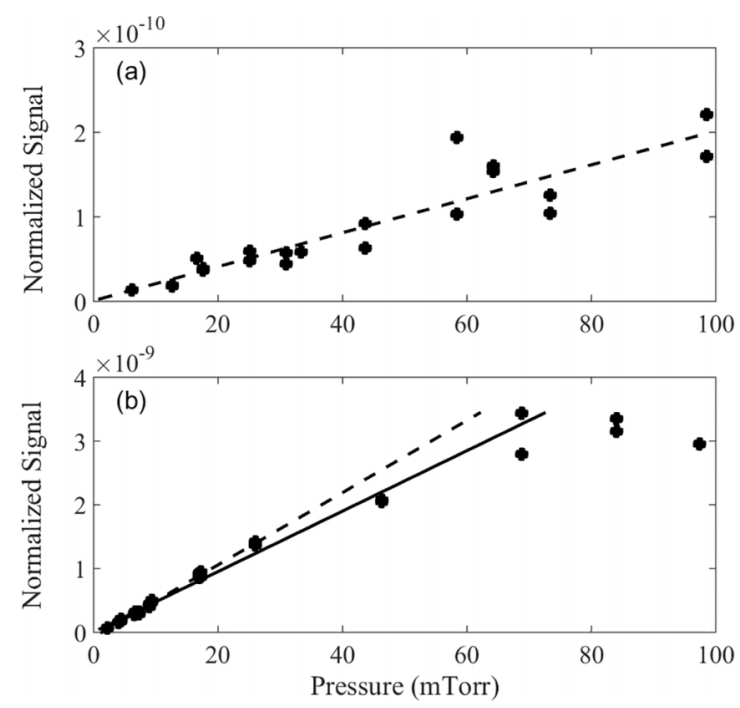

FIG. 4. Normalized TALIF signal for (a) xenon and (b) krypton as a function of neutral gas pressure. 


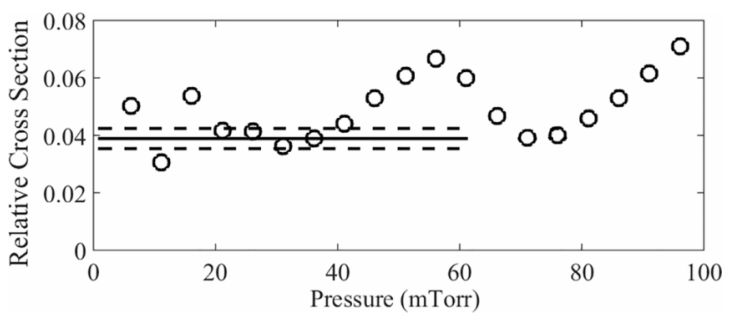

FIG. 5. The absorption cross section ratio for xenon to krypton. Dashed lines represent the ratio of fits from Fig. 4, and solid is their average.

\section{KRYPTON AND XENON MEASUREMENTS}

The total, integrated over wavelength, TALIF signal normalized by the square of the laser energy, the detector gain, and the wavelength dependent PMT quantum efficiency is therefore directly proportional to the neutral gas density. Examples of both krypton and xenon TALIF spectra are shown in Figure 3. To ensure that the two measurements employed the exact same optical path and collection volumes, two fixed irises were placed along the optical path and the injection beam constrained to pass through both irises at all times (see Figure 2).

The normalized TALIF signals for both krypton and xenon are shown in Figure 4 as a function of neutral gas pressure. Note that in these units, the xenon absorption cross section is significantly smaller than the krypton absorption cross section. At low gas pressures, both sets of measurements are linear with increasing neutral pressure. At the largest pressures investigated, the krypton TALIF signal diverges from linearity, most likely as a result of increased absorption of the laser light along the injected beam path. That the krypton data saturates at the highest pressures is consistent with a krypton absorption cross section that is much larger than the xenon cross section. Shown in Figure 4 are linear fits to the TALIF measurement. For krypton, two different linear fits are shown; one that includes the measurements up to $60 \mathrm{mTorr}$ (solid line) and one that stops at $50 \mathrm{mTorr}$ (dashed line). The slope of the linear fit is proportional to the absorption cross section. The uncertainty in the pressure measurement is \pm 1.5 mTorr and the linear fits for both species cross the pressure axis within the range of pressure uncertainty.

Shown in Figure 5 is the ratio of the measured krypton and xenon two-photon absorption cross sections as a function of neutral gas pressure. The dashed lines correspond to the ratio of the linear fits using the two different krypton fits. The solid line is the average of the two krypton fits. Using the ratio of the fits, the relative cross section is $0.038 \pm 0.005$. The average of the ratio measurements for pressures less than 50 mTorr yields a relative cross section of $0.041 \pm 0.008$. Using the krypton to hydrogen two photo absorption ratio of 0.62 , we obtain a relative two-photon absorption cross section between xenon and hydrogen of $0.024 \pm 0.001$.

\section{SUMMARY}

These measurements confirm that this xenon TALIF scheme is a viable calibration method for hydrogen TALIF. The similarity in excitation and fluorescence wavelengths with

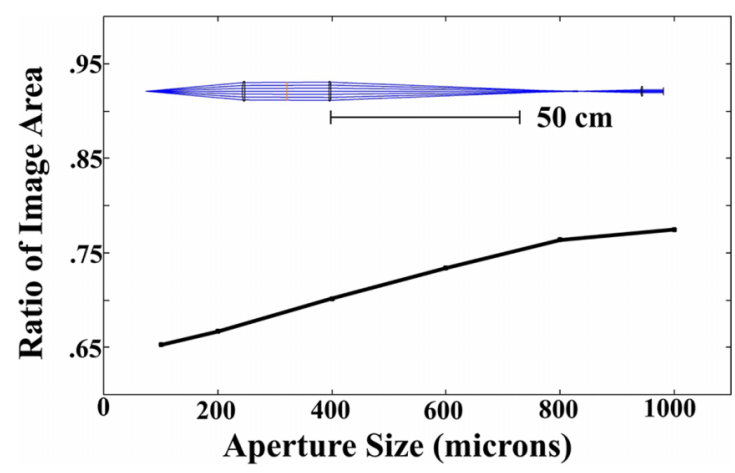

FIG. 6. Ratio of the effective areas of the image spot size for $826 \mathrm{~nm}$ and $656 \mathrm{~nm}$ light after passing through a spatial filter of varying diameter. Inset is the Zemax ${ }^{\mathrm{TM}}$ model of the confocal configuration including a spatial filter that was used for these calculations.

the hydrogen TALIF scheme eliminates chromatic differences in the collection optics that impede calibration and alignment using the typical krypton scheme. Note that the uncertainty reported here in the xenon to hydrogen absorption cross section ratio does not include the effect of the uncertainty in the krypton to hydrogen cross section ratio in the literature.

In recent deuterium TALIF experiments on the HIT-SI3 spheromak, high quality krypton TALIF signals were obtained after optimizing the collection optics for krypton TALIF emission. However, after optimizing for krypton TALIF emission, the deuterium TALIF signal vanished. The lack of deuterium signal was traced to the focal-plane defining pinhole in the TALIF emission collection optical path. When optimized for $826 \mathrm{~nm}$ fluorescence, the pinhole served as an effective blocker of $656 \mathrm{~nm}$ deuterium fluorescence. The impact of the differing fluorescent wavelengths is demonstrated with the Zemax ${ }^{\mathrm{TM}}$ model of a TALIF optical system shown in Figure 6. For a light collection path of 1-2 meters, as is typical of TALIF designs for fusion systems, the Zemax ${ }^{\mathrm{TM}}$ model shows significant blockage of the $656 \mathrm{~nm}$ light when the system is optimized for the $826 \mathrm{~nm}$ krypton TALIF fluorescence.

\section{ACKNOWLEDGMENTS}

This work was supported by Department of Energy Award No. DE-SC0004736 and NSF Award No. PHYS-1360278.

${ }^{1}$ H. Q. Wang et al., Nucl. Fusion 54, 124001 (2014).

${ }^{2}$ D. J. Battaglia et al., Nucl. Fusion 53, 113032 (2013).

${ }^{3}$ JET EFDA Contributors et al., J. Nucl. Mater. 463, 450 (2015).

${ }^{4}$ R. M. Magee, M. E. Galante, D. McCarren, E. E. Scime, R. L. Boivin, N. H. Brooks, R. J. Groebner, D. N. Hill, and G. D. Porter, Rev. Sci. Instrum. 83, 10D701 (2012).

${ }^{5}$ M. R. Galante, M. Magee, and E. Scime, Phys. Plasmas 21, 055704 (2014).

${ }^{6}$ C. C. Klepper, T. E. Evans, G. Haas, G. L. Jackson, and R. Maingi, J. Vac. Sci. Technol., A 11, 446 (1993).

${ }^{7}$ M. Stamp, K. Behringer, M. Forrest, P. Morgan, and H. Summers, J. Nucl. Mater. 162, 404 (1989).

${ }^{8}$ H. P. Summers et al., Plasma Phys. Controlled Fusion 48, 263 (2006).

${ }^{9}$ C. M. Samuell and C. S. Corr, Plasma Sources Sci. Technol. 25, 015014 (2016).

${ }^{10}$ K. Niemi, V. Schultz von Gathen, and H. F. Döbele, J. Phys. D 34, 2330 (2001).

${ }^{11}$ J. Bokor, R. Freeman, J. White, and R. Storz, Phys. Rev. A 24, 612 (1981).

${ }^{12}$ E. B. Saloman, J. Phys. Chem. Ref. Data 33(3), 765 (2004).

${ }^{13}$ L. Liard, A. Aanesland, and P. Chabert, J. Phys. D: Appl. Phys. 45, 235201 (2012).

${ }^{14}$ T. R. Jarboe et al., Fusion Sci. Technol. 66, 369 (2014). 